\title{
Interactions and Molecular Docking Studies of Cefonicid Sodium with Papain Amino Acid Residues
}

\author{
Xu Cheng, Baosheng Liu*, Hongcai Zhang, Chundan Wang \\ College of Chemistry \& Environmental Science, Hebei University, Baoding, China \\ Email address: \\ lbs@hbu.edu.cn (Baosheng Liu) \\ ${ }^{*}$ Corresponding author
}

\section{To cite this article:}

Xu Cheng, Baosheng Liu, Hongcai Zhang, Chundan Wang. Interactions and Molecular Docking Studies of Cefonicid Sodium with Papain Amino Acid Residues. International Journal of Computational and Theoretical Chemistry. Vol. 7, No. 1, 2019, pp. 14-21.

doi: $10.11648 /$ j.ijctc. 20190701.13

Received: January 17, 2019; Accepted: February 25, 2019; Published: March 20, 2019

\begin{abstract}
This study aims to investigate the interaction between cefonicid sodium (CFS) and papain (PAPA) using fluorescence spectroscopy, synchronous fluorescence spectroscopy and molecular docking methods. The results indicated that the fluorescence intensity of PAPA was decreased considerably upon the addition of CFS through a static quenching mechanism. Synchronous fluorescence spectroscopy studies showed that the combination of CFS and PAPA could change the conformation of PAPA. At the temperature of $293 \mathrm{~K}$, there was a good linear relationship between the fluorescence intensity of the system and the concentration of CFS in the range of $6.0 \times 10^{-6}$ to $1.0 \times 10^{-4} \mathrm{~mol} / \mathrm{L}$ and the detection limit of the method was $3.05 \times 10^{-6} \mathrm{~mol} / \mathrm{L}$ $(n=10)$. From the results of the thermodynamic constant and molecular model analysis, it could be inferred that the CFS and PAPA molecules mainly combine by electrostatic attraction and hydrogen bonding. The binding model was established based on the experimental data, and the binding rate data of CFS and PAPA was obtained. The results showed that taking PAPA while taking CFS was safe.
\end{abstract}

Keywords: Cefonicid Sodium, Papain, Spectroscopy, Molecular Docking, Combination Rate

\section{Introduction}

Papain (PAPA) consists of a single peptide chain with a molecular weight of 23406 and is composed of about 212 amino acid residues [1]. The isoelectric point of PAPA is 8.75 , which can produce a role in a neutral or acidic environment and is most suitable for a $\mathrm{pH}$ of 6 to 7 (generally 3 to 9.5). PAPA has high heat resistance and is not completely inactivated at $90^{\circ} \mathrm{C}$. The optimum temperature is 55 to $65^{\circ} \mathrm{C}$ (normally 10 to $85^{\circ} \mathrm{C}$ ). PAPA is less expensive and cheaper than microbial enzymes. It is widely found in roots, stems, leaves, and fruits of papayas [2], which is the most abundant in immature milk. The active center of PAPA contains cysteine and belongs to thiol protease and it has the characteristics of high enzyme activity, good thermal stability, and natural health and safety. PAPA is widely used in the food industry. In the food industry, PAPA is mainly used for cold resistance of beer (hydrolyzing proteins in beer to avoid turbidity caused by refrigeration) [3], meat softening (hydrolysis of muscle protein and collagen, softening of meat) [4]. PAPA also helps precooking cereals, hydrolyzes protein, and produces meat flavors. In addition, PAPA is used to hydrolyze soybeans and concentrate protein. The modified soybean concentrated protein can be applied to food processing industries such as meat products, dairy products and beverages. Therefore, PAPA is one of the most researched, widely used and highly promising natural plant proteases in the food industry all over the world [5].

The structure of CFS was shown in Figure 1. CFS is the second-generation cephalosporin antibiotic drug [6]. CFS is a broad-spectrum cephalosporin antibiotic that exerts its antibacterial activity by inhibiting bacterial cell wall synthesis [7]. So far, there has been no report on the interaction between CFS and PAPA. In addition to acting on the human body, CFS is also widely used for septicemia in pigs, bone and joint infections, etc. CFS can treat poultry infectious diseases such as respiratory infections, urinary tract infections, etc. CFS has a positive effect on disease treatment with livestock. Due to the extensive use of CFS and PAPA, it will cause its residues in various food products such as meat products and dairy 
products. Therefore, it is particularly important to study the interaction mechanism between PAPA and CFS. So far, relevant reports have not been reported. In this study, fluorescence spectroscopy and molecular docking is used to study the interaction between PAPA and CFS, focusing on the interaction constants, the number of binding sites, the thermodynamic parameters and the optimal conformation of the interaction between the PAPA and CFS. According to the experimental data, the binding rate of CFS-PAPA system was calculated, and the safety of simultaneous intake of CFS and PAPA was explored.

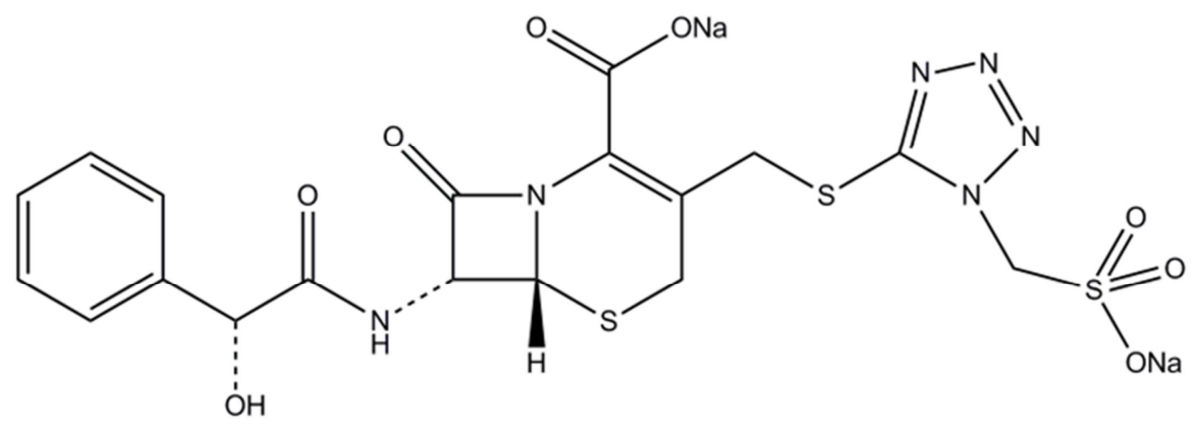

Figure 1. Chemical structure of CFS

\section{Experimental}

\subsection{Apparatus and Materials}

\subsubsection{Apparatus}

All fluorescence spectra were recorded with a Shimadzu RF-5301PC Spectro fluorophotometer. All pH measurements were carried out with a pHS-3C precision acidity meter (Leici, Shanghai). All temperatures were controlled by SYC-15B Super Constant Temperature Bath (Nanjing Electronic Equipment Factory).

\subsubsection{Materials}

The standard of CFS (CAS\#, 64485-93-4) was formulated to a stock concentration of $5.0 \times 10^{-4} \mathrm{~mol} / \mathrm{L}$ and diluted to the required concentration during the experiment. PAPA (The purity of PAPA $\geq 99 \%$, Sigma) was formulated to a stock concentration of $4.0 \times 10^{-4} \mathrm{~mol} / \mathrm{L}$. Tris- $\mathrm{HCl}$ buffer solution containing $\mathrm{NaCl}(0.15 \mathrm{~mol} / \mathrm{L})$ was used to maintain the $\mathrm{pH}$ of the solution at 7.40. All other reagents and solvents were all of analytical reagent grade. Double distilled water was used throughout the experiments and the required solution was stored in dark at $277 \mathrm{~K}$.

The fluorescence intensities were corrected for absorption of exciting light and reabsorption of the emitted light to decrease the inner filter effect using the Eq. (1) [8]:

$$
I_{c o r}=I_{o b s} \times e^{\left(A_{e x}+A_{e m}\right) / 2}
$$

Where $I_{c o r}$ and $I_{o b s}$ are the fluorescence intensities corrected and observed, respectively, and $A_{e x}$ and $A_{e m}$ are the absorption of the system at the excitation and the emission wavelength, respectively. The intensity of fluorescence used in this paper is the corrected fluorescence intensity.

\subsection{Experimental Procedure}

\subsubsection{Fluorescence Measurements}

$1.0 \mathrm{~mL}$ Tris- $\mathrm{HCl}$ buffer solution, $1.0 \mathrm{~mL} 6.0 \times 10^{-5} \mathrm{~mol} / \mathrm{L}$ PAPA solution and different volumes of CFS solution were added to a series of $10.0 \mathrm{~mL}$ colorimetric tubes. The samples were diluted to scaled volume with double-distilled water, mixed thoroughly by shaking and kept static for $30 \mathrm{~min}$ at different temperatures $(293,308$ and $318 \mathrm{~K})$. Fluorescence spectra were recorded on a spectro fluorometer equipped with a xenon lamp source and a $1.0 \mathrm{~cm}$ quartz cell. Fluorescence quenching spectra were measured over the range $280-450 \mathrm{~nm}$ with an excitation wavelength of $280 \mathrm{~nm}$ or $295 \mathrm{~nm}$ at three temperatures (293, 308 and 318 K). Synchronous fluorescence spectra were obtained by setting the excitation and emission wavelength intervals, $\Delta \lambda$, at 15 and $60 \mathrm{~nm}$. The widths of the excitation and emission slits were set to $5.0 \mathrm{~nm}$.

\subsubsection{Molecular Docking}

The two-dimensional structure of CFS was drawn in ChemDraw 15.0 and its three-dimensional structure was optimized by model of molecular mechanics in ChemDraw 3D. The crystal structure of PAPA used for molecular docking was obtained from protein data bank (PAPA ID: 1KHP) [9]. Waters and all other HETATM molecules were removed from the PAPA PDB file. Polar hydrogen atoms and Gasteiger charges were added to prepare the PAPA molecule for docking. Protein-ligand docking was performed with the rigid docking tool in the Autodock 4.2.6. In this work, the selection of flexible residues for the induced fit is based on the active site of the PAPA. The most favorable docking model was selected according to the binding energy and the geometry matching [10].

\section{Results and Discussion}

\subsection{Fluorescence Spectra of CFS-PAPA System}

For the same protein, the fluorescence effect is generated by the chromophores of tryptophan (Trp), tyrosine (Tyr) and phenylalanine (Phe) residues [11]. At $280 \mathrm{~nm}$ wavelength, the Trp and Tyr residues in protein are excited, whereas at 295 $\mathrm{nm}$ wavelength, only Trp residues are excited [12]. When the excitation wavelength was $280 \mathrm{~nm}$ (or $295 \mathrm{~nm}$ ), PAPA had a 
strong fluorescence emission peak at $347 \mathrm{~nm}$ (or $332 \mathrm{~nm}$ ) (No fluorescence was observed in CFS solution under experimental conditions [13]). The fluorescence intensity of PAPA decreased regularly with the addition of CFS when the excitation wavelength was $280 \mathrm{~nm}$ (it was similar to $295 \mathrm{~nm}$ ), which showed that there was a interaction between CFS and PAPA. To confirm the quenching mechanism, the fluorescence quenching data were analyzed using the Stern-Volmer Eq. (2) [14, 15]. We listed the results obtained in Table 1.

$$
\boldsymbol{I}_{0} / \boldsymbol{I}=1+\boldsymbol{K}_{q} \tau_{0}[\boldsymbol{L}]=1+\boldsymbol{K}_{s v}[L]=1+\boldsymbol{K}_{D}[L]
$$

In the Eq. (2), $I_{0}$ and $I$ are the fluorescence intensities of papain in the absence and presence of quencher, $K_{q}$ is the quenching rate constant, $\tau_{0}$ is the average lifetime of the bimolecular in the absence of quencher, $K_{S V}$ is the Stern-Volmer quenching constant, and $[L]$ is the concentration of quencher. Dynamic and static quenching can be distinguished by the temperature dependence of the quenching. The values of $K_{S V}$ decrease with an increasing temperature for static quenching and the reverse result for dynamic quenching
$[16]$.

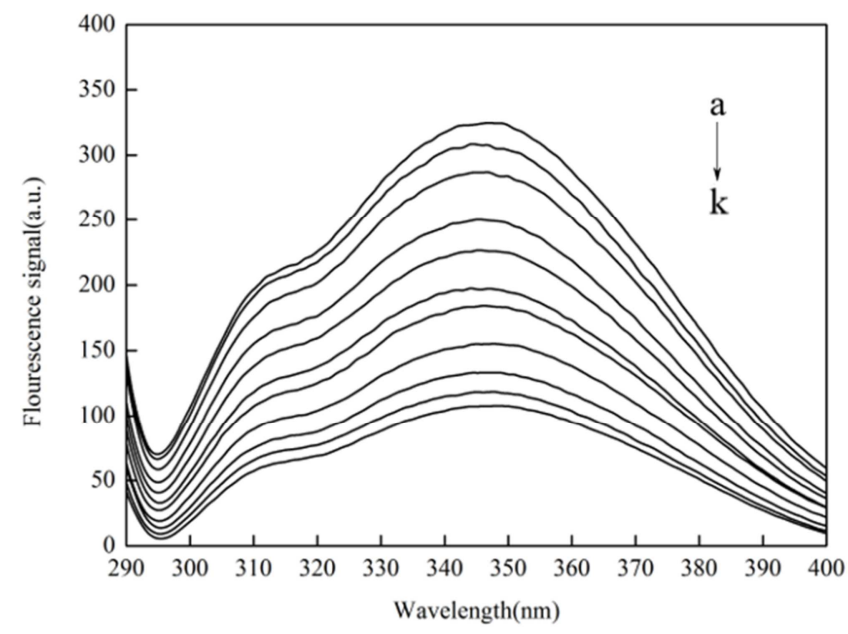

Figure 2. Fluorescence spectrum of CFS-PAPA system $\left(\lambda_{e x}=280 \mathrm{~nm}, T=293\right.$ $K)$.

$C_{\mathrm{PAPA}}=6.0 \times 10^{-6} \mathrm{~mol} / \mathrm{L} ; 1 \sim 10 C_{\mathrm{CFS}}=(0,1.0,2.0,3.0,4.0,5.0,6.0,7.0,8.0,9.0$, $10.0) \times 10^{-5} \mathrm{~mol} / \mathrm{L}$

Table 1. Quenching reactive parameters of CFS-PAPA at different temperatures.

\begin{tabular}{|c|c|c|c|c|c|c|c|}
\hline System & $T /(\mathbf{K})$ & $K_{q} /\left(\mathrm{L} \cdot \mathrm{mol}^{-1} \cdot \mathrm{s}^{-1}\right)$ & $K_{s v} /\left(\mathbf{L} \cdot \mathrm{mol}^{-1}\right)$ & $r_{1}$ & $K_{a} /\left(\mathbf{L} \cdot \mathrm{mol}^{-1}\right)$ & $n$ & $r_{2}$ \\
\hline \multirow{3}{*}{$\lambda=280$} & 293 & $1.38 \times 10^{12}$ & $1.38 \times 10^{4}$ & 0.9913 & $1.27 \times 10^{4}$ & 0.99 & 0.9938 \\
\hline & 308 & $1.20 \times 10^{12}$ & $1.20 \times 10^{4}$ & 0.9955 & $1.21 \times 10^{4}$ & 1.06 & 0.9997 \\
\hline & 318 & $7.40 \times 10^{11}$ & $7.40 \times 10^{3}$ & 0.9975 & $1.04 \times 10^{4}$ & 0.86 & 0.9825 \\
\hline \multirow{3}{*}{$\lambda=295$} & 293 & $1.16 \times 10^{12}$ & $1.16 \times 10^{4}$ & 0.9933 & $1.16 \times 10^{4}$ & 0.98 & 0.9929 \\
\hline & 308 & $1.01 \times 10^{12}$ & $1.01 \times 10^{4}$ & 0.9892 & $9.61 \times 10^{3}$ & 1.04 & 0.9965 \\
\hline & 318 & $9.28 \times 10^{11}$ & $9.28 \times 10^{3}$ & 0.9992 & $6.51 \times 10^{3}$ & 1.01 & 0.9983 \\
\hline
\end{tabular}

$r_{l}$ is the linear relative coefficient of $F_{0} / F \sim[Q] ; r_{2}$ is the linear relative coefficient of $\lg \left[\left(F_{0}-F\right) /\right] \sim \lg \left\{[L]-n\left[B_{t}\right]\left(F_{0}-F\right) / F_{0}\right\}$

In Table 1, the Stern-Volmer quenching constants at different temperatures $(293,308$ and $318 \mathrm{~K})$ were presented. The results showed that $K_{q}$ decreased with the increase in temperature, which indicated that the quenching mechanism between PAPA and CFS was a static type. In addition, all the values of $K_{q}$ were much greater than the maximum scatter collision quenching constant of various quenchers $\left(2 \times 10^{10}\right.$ $\left.\mathrm{L} \cdot \mathrm{mol}^{-1} \cdot \mathrm{s}^{-1}\right)$. It showed that CFS and PAPA reacted to form CFS-PAPA complex [17].

$$
\lg \left(\frac{I_{0}-I}{I}\right)=n \lg K_{a}+\operatorname{nlg}\left\{[L]-n \frac{I_{0}-I}{I_{0}}\left[B_{t}\right]\right\}
$$

The relationship between fluorescence intensity and the concentration of quencher can usually be described using Eq. (3) to obtain the binding constant $\left(K_{a}\right)$ and the number of binging sites $(n)$ : where $[L]$ and $\left[B_{t}\right]$ are the total concentrations of CFS and PAPA, respectively. Use Eq. (3) to further calculate binding constants and binding sites. From Table 1 , it could be seen that $K_{a}$ decreased with increasing temperature, indicating that CFS had a static quenching of PAPA fluorescence. The results showed that all the values of $n$ were approximately equal to 1 at different temperatures, implying that there was just one binding site for CFS existing in PAPA [18, 19]. Besides, the value of $K_{a}$ was far greater when wavelength was $280 \mathrm{~nm}$ than that when wavelength was
$295 \mathrm{~nm}$ at the same temperature, indicating that the binding site between CFS-PAPA originated from the Trp and Tyr residues of PAPA.

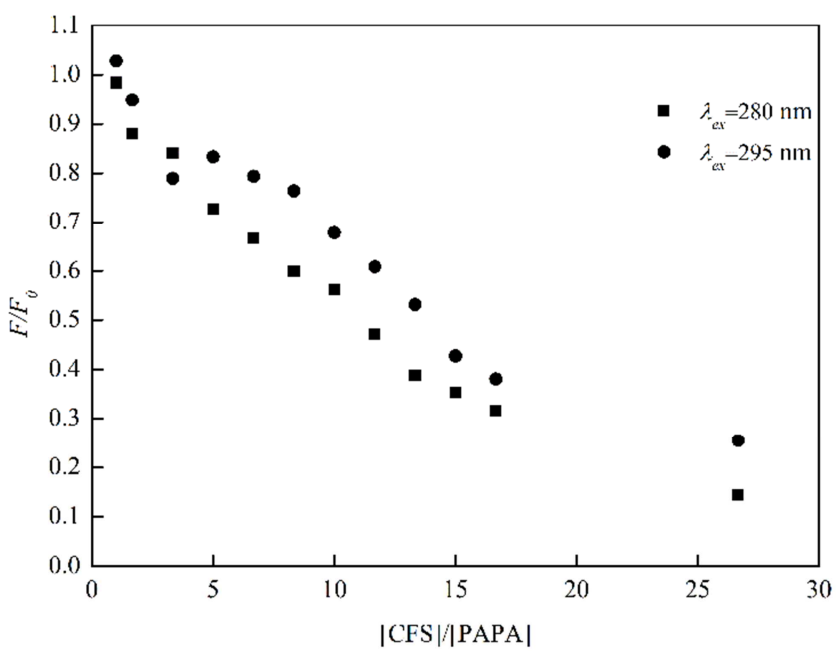

Figure 3. Fluorescence quenching curves of CFS-PAPA system at $\lambda_{e x}=280$ $\mathrm{nm}, \lambda_{e x}=295 \mathrm{~nm}(\mathrm{~T}=293 \mathrm{~K})$.

$C_{\mathrm{PAPA}}=6.0 \times 10^{-6} \mathrm{~mol} / \mathrm{L}, 1 \sim 12: C_{\mathrm{CFS}}=(0.6,1.0,2.0,3.0,4.0,5.0,6.0,7.0,8.0$, $9.0,10.0,16.0) \times 10^{-5} \mathrm{~mol} / \mathrm{L}$

The reaction of amino acid residues can be realized at the 
level of amino acid residues and the reaction mechanism between drugs and proteins can be further revealed. The data in Figure 3 could infer the involvement of Trp and Tyr residues in the CFS-PAPA system and the extent of the reaction. Figure 3 showed that the quenching at $280 \mathrm{~nm}$ was much greater than that at $295 \mathrm{~nm}$, indicating that both Trp and Tyr residues of PAPA participate in the CFS-PAPA reaction. In Table 1, $K_{a(\lambda e x=280 \mathrm{~nm})}$ was greater than $K_{a}(\lambda e x=295 \mathrm{~nm})$, it showed that both Trp and Tyr residues of PAPA participated in reaction.

\subsection{Synchronous Fluorescence Spectral Studies of CFS-PAPA System}
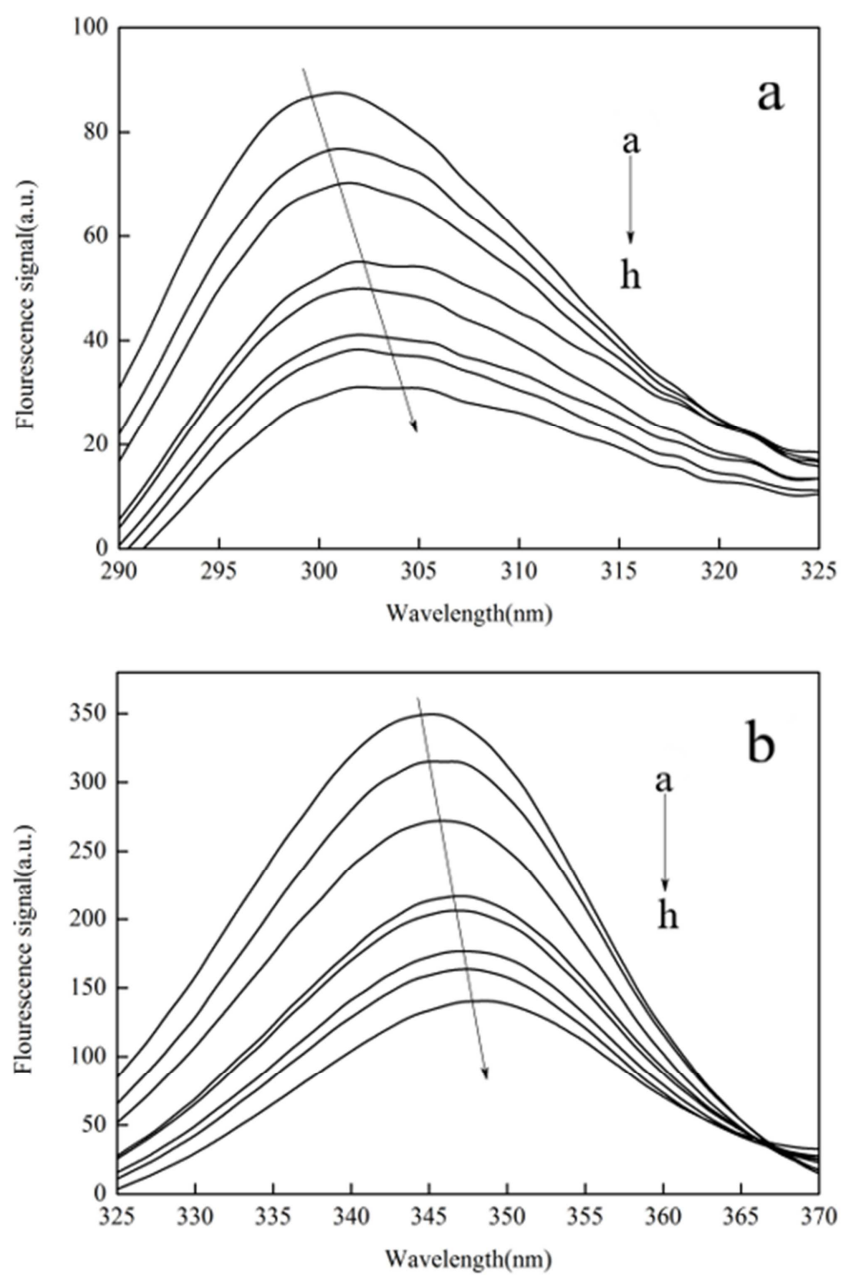

Figure 4. Synchronous fluorescence spectrum of CFS-PAPA (T=293 K) (a) $\Delta \lambda=15 \mathrm{~nm}$; (b) $\Delta \lambda=60 \mathrm{~nm}$.

$C_{\mathrm{PAPA}}=6.0 \times 10^{-6} \mathrm{~mol} / \mathrm{L} ; 1 \sim 8 C_{\mathrm{CFS}}=(0,2.0,3.0,5.0,6.0,7.0,8.0,9.0) \times 10^{-5}$ $\mathrm{mol} / \mathrm{L}$

For synchronous fluorescence spectra of proteins, when the $\Delta \lambda$ value between the excitation and emission wavelengths is stabilized at 15 or $60 \mathrm{~nm}$, the synchronous fluorescence gives characteristic information for Tyr or Trp residues, respectively $[20,21]$. Synchronous fluorescence spectra of Tyr residues and Trp residues in PAPA after addition of different concentrations of CFS were shown in Figure 4. From Figure 4, it could be concluded that the fluorescence intensity of Tyr residues and Trp residues of PAPA decreased regularly as the concentration of CFS increased. This conclusion showed that both Tyr residues and Trp residues of CFS interacted with PAPA, which was the same as the fluorescence spectra. When $\Delta \lambda$ was 15 or $60 \mathrm{~nm}$, a gradual decrease in the fluorescence intensity of PAPA and a slight redshift at the maximum emission wavelength were observed upon addition of CFS. This indicated that the microenvironment of Tyr and Trp residue was both changed, polarity around the Trp and Tyr residues increased, hydrophobicity decreased [22], extension degree of the peptide chain increased, and structure of proteins became loose [23].

\subsection{The Standard Curve of Interaction Between CFS-PAPA System}

According to the experimental method, the concentration of PAPA was fixed at $293 \mathrm{~K}$, different concentrations of CFS were added to it, and the relationship between fluorescence intensity $\Delta I\left(\Delta I=I_{0}-I\right)$ and CFS concentration $c$ was investigated. The result showed that within the range of certain concentration $\left(4.0 \times 10^{-6} \sim 1.0 \times 10^{-4} \mathrm{~mol} / \mathrm{L}\right)$, the fluorescence intensity of CFS-PAPA system had a good linear relationship with concentration of CFS. The linear regression equation was: $\Delta I=2.26 \times 10^{6} \mathrm{C}(\mathrm{mol} / \mathrm{L})+11.27$, and the correlation coefficient $r=0.9915$. And the detection limit of the method was $3.05 \times 10^{-6} \mathrm{~mol} / \mathrm{L}(n=10)$. It was shown that the fluorescence quenching reaction of PAPA on CFS could be used to achieve a rapid determination of the CFS content in actual drugs.

\subsection{Cooperativity of CFS-PAPA System}

In biochemistry, the binding of a ligand molecule at one site of a macromolecule often influences the affinity for other ligand molecules at additional sites. This is known as cooperative binding. It is classified into positive cooperativity, negative cooperativity and non-cooperativity according to the promotion or inhibition of the affinity for other ligand molecules. Hill's coefficient provides a way to quantify this effect and is calculated graphically on the basis of the following Eq. (4) [24]:

$$
\lg \{Y /(1-Y)\}=\lg K_{a}+n_{H} \lg [L]
$$

Where, $Y$ is the fractional binding saturation. $K_{a}$ is the binding constant, $n_{H}$ is the Hill's coefficient and $[L]$ is the concentration of CFS. $n_{H}>1$ indicate positive cooperativity, $n_{H}<1$ indicate negative cooperativity and $n_{H}=1$ indicate a non-cooperative reaction [25].

For fluorescence measurement:

$$
\begin{gathered}
Y /(1-Y)=Q /\left(Q_{m}-Q\right) \\
Q=1-I / I_{0}
\end{gathered}
$$

Where $1 / Q_{m}$ is intercept of the plot $1 / Q$ versus $1 /[L]$. The binding of the receptor PAPA to the ligand CFS was analyze dusing Hill coefficent $n_{H}$ to compare with 1. According to Eq. 
(4), Eq. (5) and Eq. (6), we could obtain the $n_{H}$ value of CFS-PAPA system. The results were shown in Table 2. The values of $n_{H}$ were equal to 1 approximately at different temperatures, indicating that there was non-cooperative reaction between PAPA and CFS. In other words, the binding of a ligand molecule at one site of a macromolecule had no influence on the affinity for other ligand molecules at additional sites [26]. Therefore, the binding of CFS to PAPA Tyr residues and Trp residues did not affect the binding of PAPA and other ligand molecules.

Table 2. Hill coefficient of CFS-PAPA systems at different temperatures.

\begin{tabular}{lllll}
\hline \multirow{2}{*}{$\mathbf{T} / \mathbf{K}$} & \multicolumn{3}{l}{$\lambda_{\boldsymbol{e x}}=\mathbf{2 8 0} \mathbf{n m}$} & \multicolumn{2}{l}{$\boldsymbol{\lambda}_{\boldsymbol{e x}}=\mathbf{2 9 5} \mathbf{\mathbf { n m }}$} \\
\cline { 2 - 5 } & $\boldsymbol{n}_{\boldsymbol{H}}$ & $\boldsymbol{r}_{3}$ & $\boldsymbol{n}_{\boldsymbol{H}}$ & $\boldsymbol{r}_{3}$ \\
\hline 293 & 0.97 & 0.9924 & 1.09 & 0.9958 \\
308 & 1.01 & 0.9906 & 1.10 & 0.9987 \\
318 & 0.96 & 0.9962 & 1.03 & 0.9955 \\
\hline
\end{tabular}

$n_{H}$ is Hill's coefficient, $r_{3}$ is the linear relative coefficient of $\lg [Y /(1-Y)]$ $\sim \lg [L]$.

\subsection{Thermodynamic Parameters}

In general, the interaction force between the small drug molecule and biological macromolecule includes hydrogen bond, Vander Waals force, electrostatic interactions, and hydrophobic force [27]. These interactions are important to understand the binding mode of protein-ligand complexes. The type of force of the binding reaction can be judged based on thermodynamic parameters [28]. When enthalpy change $(\Delta H)$ does not fluctuate considerably in the temperature range considered, both enthalpy change $(\Delta H)$ and entropy change $(\Delta S)$ can be obtained from Van's Hoff equation. $\Delta G$ could be calculated from Eq. (8) [29]. Calculate $\Delta G, \Delta S$ and $\Delta H$ according to Eq. (7), Eq. (8) and the results were shown in Table 3.

$$
\begin{aligned}
& R \ln K=\Delta S-\Delta H / T \\
& \Delta G=\Delta H-T \Delta S
\end{aligned}
$$

\begin{tabular}{|c|c|c|c|c|c|}
\hline System & $T /(\mathbf{K})$ & $K_{a} /\left(\mathrm{L} \cdot \mathrm{mol}^{-1}\right)$ & $\Delta H /\left(\mathrm{kJ} \cdot \mathrm{mol}^{-1}\right)$ & $\Delta S /\left(\mathrm{J} \cdot \mathrm{mol}^{-1} \cdot \mathrm{K}^{-1}\right)$ & $\Delta G /\left(\mathrm{kJ} \cdot \mathrm{mol}^{-1}\right)$ \\
\hline \multirow{3}{*}{$\lambda_{e x}=280 \mathrm{~nm}$} & 293 & $1.27 \times 10^{4}$ & \multirow{3}{*}{-5.826} & 58.68 & -23.02 \\
\hline & 308 & $1.21 \times 10^{4}$ & & 59.25 & -24.07 \\
\hline & 318 & $1.04 \times 10^{4}$ & & 58.58 & -24.45 \\
\hline
\end{tabular}

Table 3. The thermodynamic parameters of CFS-PAPA system at different temperatures.

Generally speaking, four types of interactions including hydrogen bonding, Vander Waals interactions, electrostatic forces and hydrophobic interactions are the major binding forces affecting the interaction of different substances with macro biomolecules. From the thermodynamic point of view, $\Delta S>0$ and $\Delta H<0$ approves an electrostatic force, $\Delta S>0$ and $\Delta H>0$ proposes a hydrophobic interaction, and $\Delta S<0$ and $\Delta H<0$ indicates the vander Waals force or hydrogen bond formation [30]. From Table 3, it could be seen that $\Delta G<0, \Delta S>$ 0 , indicating that the quenching reaction of CFS on PAPA proceed spontaneously. From Table 3 we could also know that $\Delta H<0, \Delta S>0$, indicating the combination of electrostatic attraction between CFS and PAPA.

\subsection{Molecular Docking}

Molecular docking is an important technique in structural molecular biology and drug discovery with the ability to study the exact binding location of ligands on protein. To further illustrate the interaction mechanism of CFS and PAPA we use molecular simulation technology to further explore this process. The molecular docking results showed that the binding energy of CFS and PAPA $\Delta G$ is $-5.33 \mathrm{kcal} \mathrm{mol}^{-1}$ $\left(-22.29 \mathrm{~kJ} \cdot \mathrm{mol}^{-1}\right)$. This result and the experimental results $\left(-23.02 \mathrm{~kJ} \cdot \mathrm{mol}^{-1}\right)$ were the same, showing that this binding mode could truly reflect the reaction between CFS and PAPA This difference may be due to exclusion of the solvent in docking simulations or rigidity of the receptor other than Trp and Tyr residues [31]. The optimum binding mode and site were displayed in Figure 5(a) and the amino acid residues surrounding CFS were captured. CFS was surrounded by amino acid residues of Asp158, Val157, Gly65, Ala160, Val133, Cys25, Ser205, Tyr67, Pro68, Trp26 and Gly66. Tyr67 and Trp26 are close to CFS, indicating that CFS can effectively quench the fluorescence of Trp and Tyr residues, which is consistent with the results of fluorescence spectroscopy. It could be seen from Figure 5(b) that the hydrogen bond between CFS and Tyr67 was $2.187 \AA$ and the hydrogen bond between CFS and Gly66 was $1.773 \AA$. The results indicated that there was hydrogen bonding between CFS and PAPA. From the results of molecular modeling and thermodynamic analysis, it could be inferred that the binding mode of CFS and PAPA molecules was not a single force form, but rather two intermolecular forces (electrostatic force and hydrogen bond force).

To further illustrate the molecular docking results, another PAPA conformation was selected for molecular docking experiments with CFS. Molecular docking experiments were re-examined using PAPA conformation (1KHQ). The results showed that CFS forms hydrogen bonds with Asn184 and Tyr186 residues with bond lengths of $2.238 \AA$ and $1.961 \AA$, respectively. The binding energy of this conformation to CFS is about $-24.76 \mathrm{~kJ} / \mathrm{mol}$, which is similar to the results of the spectral experiments. Therefore, the new conformation of papain is consistent with the conclusions obtained in the conformation used in the experiment. 
a

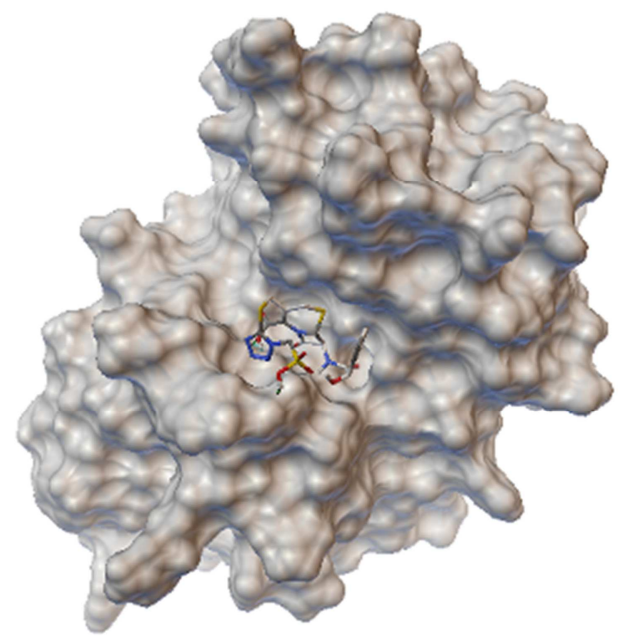

b

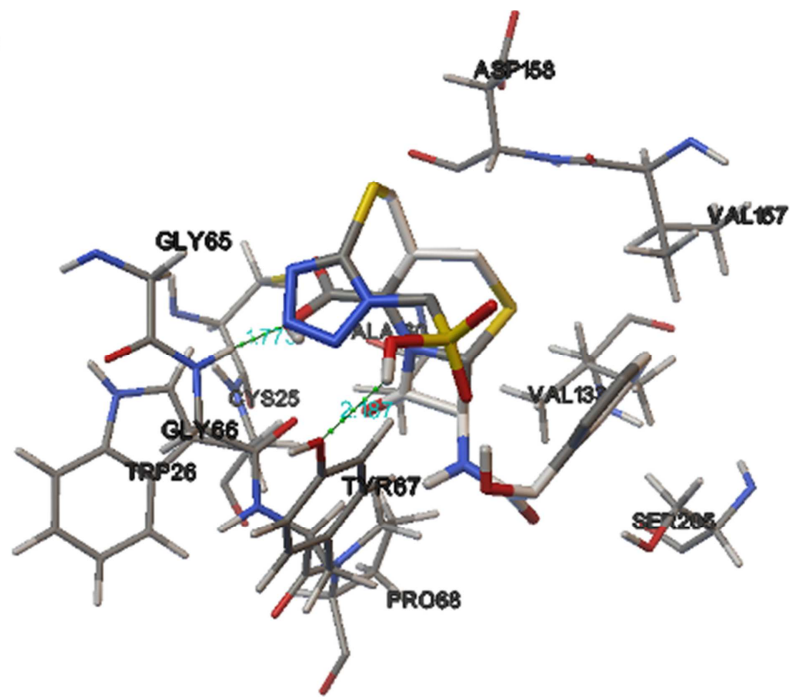

Figure 5. The interaction between CFS and PAPA. (a) An overview of the CFS molecular located in PAPA. (b) The binding mode between CFS and the defined flexible residues in the PAPA.

\subsection{The Binding Rate of CFS-PAPA System}

In the case of a CFS-PAPA binding homeostasis, one part of the protein is free-form protein and the other part is bound-form. Assumed that $n$ is the equivalent and independent number of drug binding sites, the reactions between $\operatorname{drug} Q$ and protein $B$ can be expressed as:

$$
[B]+n[Q] \rightarrow\left[B Q_{n}\right]
$$

Where $[B]$ and $[Q]$ are the free concentration of protein and colorant, respectively; $n$ is the equivalent and independent number of drug binding sites. If the interaction of $B$ with $Q$ obeyed the Langmuir single molecule adsorption model [32], the equilibrium constant $K_{a}$ can be given by following equation:

$$
\begin{aligned}
& K_{a}=\frac{\left[B Q_{n}\right]}{[Q]^{n}[B]} \quad \text { The binding rate of drug can be given by } \\
& W(Q)=\frac{x}{Q} \times 100 \%=\frac{K_{a}(Q+B)+1-\sqrt{K_{a}^{2}(Q-B)^{2}+2 K_{a}(Q+B)+1}}{2 K_{a} Q} \times 100 \%
\end{aligned}
$$

The binding rate of protein can be given by Eq. (10):

$$
W(B)=\frac{x}{B} \times 100 \%=\frac{K_{a}(Q+B)+1-\sqrt{K_{a}^{2}(Q-B)^{2}+2 K_{a}(Q+B)+1}}{2 K_{a} B} \times 100 \%
$$

The $K_{a}$ value obtained by the experiment at $\lambda_{e x}=280 \mathrm{~nm}$ was combined with the Eq. (9) and Eq. (10) to calculate the protein binding rate and drug binding rate of different concentrations of CFS at different temperatures.

The optimal consumption of human PAPA is $1.5 \mathrm{~g} /$ day, and the blood is about $10^{-6} \mathrm{~mol} / \mathrm{L}$, so it is calculated as $C_{\mathrm{PAPA}}=6.0 \times 10^{-6}$ $\mathrm{mol} / \mathrm{L}$ in this paper. According to the dose range of human injection CFS, the peak plasma concentration after intravenous infusion is $93 \mathrm{mg} / \mathrm{L} \sim 300 \mathrm{mg} / \mathrm{L}$, which is $1.59 \times 10^{-4} \sim 5.11 \times 10^{-4}$ $\mathrm{mol} / \mathrm{L}$. The $K_{a}$ value obtained by the experiment at $\lambda_{e x}=280 \mathrm{~nm}$ was combined with the Eq. (9) and Eq. (10) to calculate the drug binding rate and protein binding rate of different CFS concentrations at 293, 308 and $318 \mathrm{~K}$, respectively. The results were as follows: $W(Q)=2.50 \% \sim 1.02 \%(293 \mathrm{~K}), 2.46 \% \sim 1.01 \%$ $(308 \mathrm{~K}), 2.33 \% \sim 0.99 \%(318 \mathrm{~K}), W(B)=66.3 \% \sim 86.5 \% \quad(293 \mathrm{~K})$, $65.2 \% \sim 86.0 \%(308 \mathrm{~K}), 61.8 \% \sim 84.0 \%$ (318K). It could be seen 
from the data that both the drug binding rate and the protein binding rate decreased with increasing temperature, which was consistent with the conclusion of the fluorescence quenching experiment.

The concentration ratio of CFS to PAPA was $R=Q / B$, and the obtained data was linearly fitted. The relationship between the binding rate of $W$ and $R$ of CFS-PAPA system was plotted, as shown in Figure 6. Taking 308K data close to human body temperature as an example, when taking CFS and eating PAPA, the interaction of CFS-PAPA has little effect on the efficacy of CFS, but the free PAPA concentration is only $34.8 \% \sim 14.0 \%$. Non-linear fitting of the curve at $308 \mathrm{~K}$ yields a combined model of CFS and PAPA: $W(Q)=-0.0014 R^{2}-0.0544 R+$ $0.0116, r=0.9980, W(B)=7.7 \times 10^{-5} R^{2}-0.0033 R+0.0661, r=$ 0.9990. This data indicates that it is safe to consume PAPA during CFS administration.

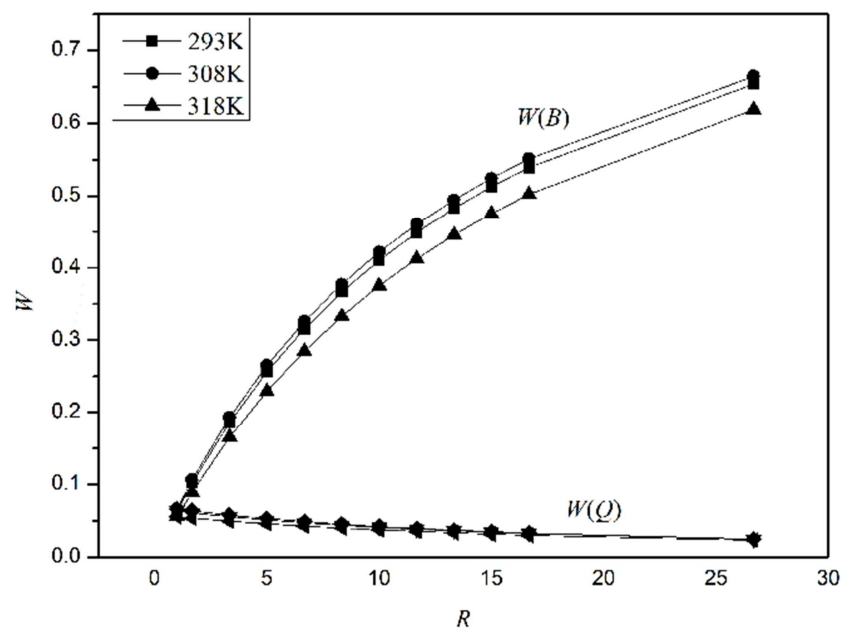

Figure 6. Protein binding rate and drug binding rate of CFS-PAPA system at different temperatures $\left(\lambda_{e x}=280 \mathrm{~nm}\right)$.

\section{Conclusions}

In this paper, the mechanism of action of CFS and PAPA was studied using fluorescence spectroscopy and synchronous fluorescence spectroscopy. Fluorescence analysis showed that the reaction between CFS and PAPA was a static quenching process. The binding parameters, binding sites and other binding parameters of CFS and PAPA reactions were obtained. The thermodynamic parameters and molecular docking results indicated that the electrostatic attraction between CFS and PAPA was the key binding force. Molecular docking also indicated that there was hydrogen bonding between them. According to the experimental data, the drug binding rate and protein binding rate of CFS-PAPA system were obtained, which provided a new idea for studying the interaction between biomacromolecules and small drug molecules.

\section{Acknowledgements}

The authors gratefully acknowledge the financial support of National Natural Science Foundation of China (No. 21375032).

\section{References}

[1] Liu XY, Zeng HY, Liao MC, Gohi BFCA and Feng B. RSC Advances, 2015, 5, 68906-68913.

[2] Wei DH, Huang XQ, Liu JJ, Tang MS, Zhan CG. Biochemistry, 2013, 52, 5145-5154.

[3] Esti M, Benucci I, Lombardelli C, Liburdi K, Garzillo AMV. Food. Bioprod. Proc, 2013, 91, 595-598.

[4] Ashie INA, Sorensen TL, Nielsen PM. Journal of Food Science, 2010, 67, 2138-2142.

[5] Zheng K, Yang H. Biological Chemical Engineering, 2017, 3, 109-110.

[6] Wang J, Xu Y, Zhang Y, Wang H, Zhong W. Rapid Communications in Mass Spectrometry, 2017, 31, 1541-1550.

[7] Duan ST, Liu BS, Li ZY, Jia X, Lu JN. Spectroscopy Letters, 2016, 49, 426-33.

[8] Steiner R F, Weinryb I. Springer US, 1971.

[9] Wang GK, Xi H, Tian F, Han MY, Lu Y. Acta Chimica Sinica, 2011, 69, 95-100.

[10] Gökoğlu E, Yılmaz E. Journal of Fluorescence, 2014, 24, 1439-1445.

[11] Zhang XF, Yang G, Dong Y, Zhao YQ, Sun XR, Chen L, Chen HB. Spectrochimica Acta Part A-Molecular and Biomolecular Spectroscopy, 2015, 137, 1280-1285.

[12] Zhang LH, Liu BS, Li ZY, Guo Y. Asian Journal of Chemistry, 2015, 30, 686-692.

[13] Zhai M, Wu HL, Zhang XH, Sun YM, Yu RQ. Science China-Chemistry, 2014, 57, 748-754.

[14] Zhao XC, Sheng F, Zheng JL, Liu RT. Journal of Agricultural \& Food Chemistry, 2011, 59, 7902-7909.

[15] Zhao XC, Liu RT, Teng Y, Liu XF. Science of the Total Environment, 2011, 409, 892-897.

[16] Jahanban-Esfahlan A, Panahi-Azar V, Sajedi S. Food Chemistry, 2016, 202, 426-431.

[17] Naveenraj S, Anandan S. Journal of Photochemistry \& Photobiology C Photochemistry Reviews, 2013, 14: 53-71.

[18] Gaudet M, Remtulla N, Jackson SE, Main ER, Bracewell DG, Aeppli G, Dalby PA. Protein Science, 2010, 19, 1544-1554.

[19] Liu BS, Xue CL, Wang J, Yang C, Zhao FL, Lv YK. Journal of Luminescence, 2010, 130, 1999-2003.

[20] Hu YY, Xu SQ, Zhu XS, Gong AQ. Spectrochimica Acta Part A Molecular \& Biomolecular Spectroscopy, 2009, 74, 526-531.

[21] Zhao XC, Lu DW, Fang H, Liu RT. Journal of Hazardous Materials, 2015, 292, 98-107.

[22] Bani-Yaseen AD. Journal of Luminescence, 2011, 131, 1042-1047.

[23] Wang YQ, Zhang HM, Zhou QH. European Journal of Medicinal Chemistry, 2009, 44, 2100-2015. 
[24] Bojko B, Sulkowska A, Maciazek-Jurczyk M, Równicka J, Sułkowski WW. Journal of Pharmaceutical \& Biomedical Analysis, 2010, 52, 384-390.

[25] Liu BS, Wang J, Xue CL, Yang C, Lv YK. Z. Phys. Chem, 2011, $225,455-468$.

[26] Khan AB, Khan JM, Ali MS, Khan RH, Kabir-ud-Din. Spectrochimica Acta Part A Molecular \& Biomolecular Spectroscopy, 2012, 97, 119-124.

[27] Cao SN, Liu BS, Li ZY, Chong BH. Journal of Luminescence, 2014, 145, 94-99.

[28] Liu YY, Zhang GW, Liao YJ, Wang YP. Spectrochimica Acta Part A Molecular \& Biomolecular Spectroscopy, 2015, 151, 498-505.
[29] Gong AQ, Zhu XS, Hu YY, Yu SH. Talanta, 2007, 73, 668-673.

[30] Iovescu A, Băran A, Stîngă G, Cantemir-Leonties AR, Maxim ME, Anghel DF. Journal of Photochemistry \& Photobiology B Biology, 2015, 153, 198-205.

[31] Jana S, Dalapati S, Ghosh S, Guchhait N. Journal of Photochemistry \& Photobiology B Biology, 2012, 112, 48-58.

[32] Liu BS, Cao SN, Li ZY, Zhong BH. Chinese Journal of Luminescence, 2013, 34, 488-493. 